\title{
Construcción del conocimiento didáctico del contenido y su transferencia a la práctica: retrato de un profesor universitario
}

\author{
Mํㅡㄹ Dolores Fernández Tilve
}

\begin{abstract}
Resumen:
El cambio profundo que está experimentado la educación superior en los últimos tiempos está teniendo una especial repercusión en los procesos de enseñar y aprender y en la formación del profesorado universitario. La aparición de una nueva estructura curricular, la propuesta de nuevos métodos de enseñanza centrados explícitamente en el aprendizaje del alumnado y la nueva concepción del trabajo del profesorado emergente tras estos cambios están generando unas exigencias pedagógico-didácticas que no tienen parangón en la reciente historia de las universidades europeas. El estudio interuniversitario que presentamos tiene como objetivo general poner de manifiesto y hacer visibles aquellas buenas prácticas docentes que realmente se centran en el alumnado y en su proceso de aprendizaje. Nuestra aproximación metodológica es de corte fenomenológico, optando por un estudio de caso múltiple. Como instrumentos de recogida de información se utilizaron la observación no participante, las entrevistas en profundidad (biográfica y de estimulación de recuerdo) y el análisis de materiales. El estudio de caso que mostramos evidencia que la importancia otorgada a la formación teórica disciplinar y a su relación con la experiencia investigadora caracterizan la construcción del Conocimiento Didáctico del Contenido (CDC), dando verdadera forma y sentido didáctico a la materia de enseñanza.
\end{abstract}

Palabras clave:

aprendizaje; conocimiento didáctico del contenido; enseñanza; profesorado; universidad. 


\title{
A didactic approach to contents and knowledge transfer to the practice: the portrait of a university professor
}

\begin{abstract}
The great change that higher education has gone through in the last few years has had specific consequences for the teaching and learning processes, as well as for teacher training. The appearance of new curricular structures, the proposal of new methods of teaching centered explicitly on student learning, and the new understanding of the work of the professor who is emerging after these changes are generating certain educational and teaching demands which compare to nothing that has existed in the recent history of European universities. The interuniversity study that we present seeks to manifest and make visible those good practices with which professors focus on the student and their learning process. Our methodological approach is of the phenomenological type, and we have opted for a multiple case study. The observation of a non-participant, in-depth interviews (biological and memory stimulation) and material analysis were used in order to collect information. The case study that we will talk about provides evidence that the importance given to the disciplinary theory education, and its relationship with research experience characterize the construction of the Pedagogical Content Knowledge (PCK), and give true form and educational meaning to the subject of teaching.
\end{abstract}

Key Words: learning; pedagogical content knowledge (PCK); professor; teaching; university.

\section{Construção do conhecimento didáctico do conteúdo e a sua transferência para prática: retrato de um professor universitário}

Resumo: A profunda mudança que o ensino superior experimentou nos últimos tempos está a ter um impacto especial no processo de ensino e aprendizagem e na formação de professores universitários. O aparecimento de uma nova estrutura curricular, a proposta de novos métodos de ensino focados explicitamente na aprendizagem dos alunos e a nova concepção do trabalho dos professores emergentes após essas mudanças geram necessidades de natureza pedagógico didáticas que não têm paralelo na história recente de Universidades europeias. O estudo interuniversitário que apresentamos tem como objetivo geral mostrar e tornar visíveis as boas práticas de ensino que realmente se focalizam nos alunos e no processo de aprendizagem. A nossa abordagem metodológica é o corte fenomenológico, optando por um estudo de caso múltiplo. Como instrumentos de recolha de informações considerámos: observação não participante, entrevistas em profundidade (estimulação biográfica e de memória) e análise de material. O estudo de caso põe em evidencia que, a importância atribuída à componente teórica das disciplinas e a sua relação com a experiência na investigação, caracterizam a construção do conhecimento didáctico do conteúdo - Knowledge Didactic Knowledge (CDC), dando verdadeira forma e significado didáctico ao tema do ensino.

Palavras-chave: aprendizagem; conhecimento didáctico do conteúdo; ensino; docentes; universidade.

\section{Construction de la Connaissance Didactique du Contenu et son transfert à la pratique: portrait d'un professeur d'université}

Résume: Le changement profond que l'enseignement supérieur a connu ces derniers temps a un impact particulier sur le processus d'enseignement et d'apprentissage et sur la formation des professeurs de luniversité. L'apparition d'une nouvelle structure curriculaire, la proposition de nouvelles méthodes d'enseignement explicitement axées sur l'apprentissage des élèves et la nouvelle conception du travail des enseignants émergents après ces changements génèrent des exigences pédagogiques et didactiques sans précédent dans l'histoire récente des Universités européennes. L'étude interuniversitaire présentée ici a pour but non seulement de mettre en valeur les bonnes pratiques d'enseignement centrées réellement sur les étudiants et leur apprentissage, mais aussi de les rendre visibles. Notre approche méthodologique est de nature phénoménologique, optant pour une étude de cas multiple. Nous avons utilisés, comme instruments de collecte d'informations, l'observation non participante, les entretiens approfondis (biographie et stimulation de la mémoire) et l'analyse des matériaux. L'étude de cas que nous présentons montre que l'importance accordée à la formation théorique disciplinaire et à sa relation avec l'expérience de recherche caractérisent la Construction de la Connaissance Didactique du Contenu (CDC), donnant une forme véritable et un sens didactique au sujet de l'enseignement.

Mots-clés: apprentissage; connaissance didactique du contenu; enseignement; personnel enseignant; université. 


\section{Introducción}

El Espacio Europeo de Educación Superior (EEES) reclama una renovación de la enseñanza y del aprendizaje universitario de tal profundidad que conforma un escenario académico inédito en las universidades españolas y que exige una docencia radicalmente distinta y de sentido opuesto a las prácticas transmisivas imperantes. La aparición de una nueva estructura curricular, la propuesta de nuevos métodos de enseñanza centrados explícitamente en el aprendizaje del alumnado y la nueva concepción del trabajo del profesorado genera unas exigencias pedagógico-didácticas importantes (Jarauta, Medina Moya y Mentado, 2016) y supone una oportunidad para impulsar la innovación e incluso re-significar el rol de la educación superior en la sociedad (Leite Ramalho y Beltrán Llavador, 2012).

En el marco de estas reflexiones, decidimos iniciar una investigación interuniversitaria que permita dar una respuesta adecuada a estas nuevas exigencias académicas y que fomenten la mejora de la calidad de la educación superior. Una investigación financiada por el Ministerio de Ciencia e Innovación (EDU 2008-05964-C03-03/EDUC), que tiene como finalidad poner de manifiesto y hacer visibles aquellas buenas prácticas docentes que realmente se centran en el alumno/a y en su proceso de aprendizaje. No pretendemos, simplemente, elaborar un listado de comportamientos docentes estandarizados y generalizables a la profesión docente universitaria, sino más bien ofrecer evidencias. Nos proponemos estudiar la enseñanza desde el conocimiento de los docentes. Para ello, nos acercamos a las experiencias, creencias, etc. que resultan útiles para la práctica. Particularmente, queremos estudiar cómo se genera y cómo opera el Conocimiento Didáctico del Contenido (CDC) en docentes noveles, iniciados y experimentados considerados altamente competentes, de las diferentes áreas de conocimiento en diferentes universidades españolas y que desarrollan su docencia bajo la lógica del EEES y fuera del mismo, en contextos presenciales y no presenciales.

Para este fin, nos centramos en los estudios sobre el conocimiento profesional del profesorado universitario, cuya repercusión en la innovación en la educación superior está todavía por explorar. En concreto, por un enfoque que se interesa por aquellos saberes disciplinares y pedagógicos, curriculares, personales, etc. que habilitan para un ejercicio competente de la enseñanza. Dentro de ese enfoque, nos hemos instalado en una línea de investigación que ha generado un poderoso y multidimensional constructo que usaremos para tratar de entender y explicar la docencia universitaria de calidad y que puede tener amplias repercusiones en la formación del profesorado universitario: el CDC, aunque toma también aspectos de los estudios sobre conocimiento práctico pues es plausible pensar que el CDC es, como se verá más adelante, estructuralmente un conocimiento que se produce "en" la práctica, "desde" la práctica y "para" la práctica. Este constructo, complejo y poliédrico, propuesto inicialmente por Shulman 
en 1983 y traducido posteriormente por Marcelo (1993), describe la capacidad de un docente para transformar pedagógicamente el conocimiento del contenido disciplinar que posee en formas y estructuras comprensibles para los estudiantes. Tal y como señala Shulman (2005), puede adquirirse de una variedad de fuentes (conocimiento del contenido científico, conocimiento del currículum, conocimiento de los alumnos, conocimiento del contexto educativo, etc.). Es un elemento central del conocimiento del profesor y resulta fundamental para promover el desarrollo profesional del profesorado, como dice el propio Acevedo (2009).

Sin embargo, esta línea de investigación se encuentra especialmente centrada en el profesorado de los niveles de educación obligatoria y a través de la comparación de profesores noveles y profesores experimentados, no así en el ámbito de la enseñanza universitaria. La literatura referida al proceso de aprendizaje en la enseñanza superior y la construcción del conocimiento profesional en el profesorado universitario es aun sorprendentemente limitada. Actualmente, en parte, como resultado de las exigencias del EEES, encontramos referencias interesantes sobre las características cognitivas y emocionales existentes en el inicio de la docencia universitaria (Cruz Tomé, 2003; Feixas, 2002); sobre el desarrollo profesional del profesor universitario (Feixas, 2002); sobre las concepciones y creencias que el docente posee acerca de la enseñanza, de los alumnos y del aprendizaje en la educación superior (Kember, 1997) y sobre la identidad profesional del profesorado universitario. No obstante, son inexistentes en nuestro país, los trabajos dedicados a explorar cómo los docentes universitarios realizan la transposición didáctica (Chevallard, 1991), proceso mediante el cual el contenido disciplinar es reorganizado y transformado teniendo en cuenta a los alumnos y alumnas, al currículo y al contexto. Pese a que empiezan a aparecer algunas obras centradas en el pensamiento del profesor universitario (Hativa y Goodyear, 2003) algunas en la identificación del "buen profesor" (Bain, 2006) y otras en la enseñanza universitaria eficaz (Knight, 2005) aún es preciso delimitar, con mayor exactitud, no tanto aquello que los docentes necesitan conocer y cómo la formación puede contribuir a su adquisición, sino indagar qué conocen los profesores universitarios y los procesos a través de los cuales adquieren ese conocimiento.

Como bien sabemos el profesorado universitario, antes que docente, es especialista en una disciplina científica concreta. Recibe, de manera inicial, formación en los contenidos científicos de su área disciplinar. Algunas investigaciones sostienen que el acercamiento a la enseñanza depende del campo disciplinar de donde provenga (Lueddeke, 2003). No obstante, la falta de una formación pedagógica sistemática no debe conducirnos a pensar que el profesorado interviene en el aula sin un cuerpo de conocimientos pedagógicos de referencia. Consciente o inconscientemente adapta, reconstruye, transforma y simplifica el contenido para hacerlo comprensible al alumnado. Esta capacidad de transformación del contenido es lo que, según Doyle (1990), 
distingue a un profesor/a de un especialista de la materia. Esa transformación se opera mediante el CDC.

En este trabajo presentamos uno de los estudios de casos realizados: un profesor iniciado o intermedio, valorado como "buen docente", perteneciente al área de las Ciencias Sociales y Jurídicas y que desarrolla su actividad de enseñanza en contextos presenciales y no presenciales.

\section{Marco metodológico}

Seleccionar un método de investigación supone optar por una serie de suposiciones que le dan fundamento. En el nivel epistemológico todo método se basa en una teoría y premisas que validan la metodología y en el nivel operacional se basa en una serie de premisas ontológicas sobre la naturaleza de los datos. La naturaleza social, simbólica y no lineal del objeto de estudio y los referentes epistemológicos que como investigadores compartimos implican que adoptemos un enfoque ontoepistémico que es ya tradicional en nuestras líneas de investigación y que tendría mucho que ver con el Interaccionismo Simbólico. Este enfoque se basa en dos conjuntos de supuestos que podríamos denominar cualitativo-fenomenológicos, ecológico-naturalistas y sistémico-complejos.

En el primer grupo de supuestos, se defiende que las prácticas humanas sólo pueden hacerse inteligibles accediendo al marco simbólico en el que las personas interpretan sus pensamientos y acciones. Comprender las acciones humanas será lo mismo que colocar nuestra atención en la vida interior y subjetiva de los actores sociales.

Desde el segundo grupo de supuestos, entendemos que las acciones humanas están parcialmente determinadas por el contexto y ambiente en el que suceden. La suposición que subyace a esta hipótesis es que los fenómenos educativos sólo pueden estudiarse en la vida real donde se producen. Sólo el contacto directo con esa realidad nos podrá garantizar el conocimiento del papel que el contexto social y cultural juega en la construcción de significados y en la comprensión que de ellos tienen las personas.

Nuestro enfoque epistemológico se concreta, metodológicamente, en un estudio de caso múltiple (Stake, 1998) en el que se usan un conjunto de herramientas intelectuales y de técnicas de recogida, análisis e interpretación de la información que detallaremos a continuación.

\subsection{Participantes, instrumentos y análisis de datos}

La muestra de profesores se elige siguiendo el procedimiento llamado por Goetz y Le Compte (1988) "selección basada en criterios" y por Patton (1987) "muestreo intencionado" que consiste en determinar las características de los sujetos que van a participar en la investigación con el fin de obtener toda la información necesaria 
para el objeto de estudio. En concreto, identificamos a 9 profesores/as universitarios reconocidos como "buenos docentes" tanto por sus pares como por los estudiantes: dominio del contenido de enseñanza, capacidad de motivar al alumnado, compromiso con la mejora de la enseñanza y obtención de buenos resultados académicos. Además de este criterio general, la selección de la muestra se realiza también en base a otros criterios más específicos como son: momento de la trayectoria profesional (novel, intermedio y experimentado) y áreas de conocimiento que actualmente regulan las titulaciones universitarias.

La observación no participante, las entrevistas en profundidad y el análisis de materiales juegan un papel central aquí. En el caso de la observación no participante de las prácticas de enseñanza, el sistema que seguimos para registrar y almacenar los datos observacionales responde a un sistema narrativo (Evertson y Green, 1989). A través de este sistema obtenemos descripciones detalladas de los fenómenos observados e identificamos patrones de conducta en situaciones específicas. Las entrevistas en profundidad (biográfica y de estimulación del recuerdo) nos sirvieron para contrastar las interpretaciones realizadas con el significado que le atribuyen los profesores/as participantes.

Por lo que respecta al análisis de datos, utilizamos el método de las comparaciones constantes propuesto por Glaser y Strauss (1967). Un método generativo, constructivo e inductivo en el que se combina la codificación inductiva de categorías con la comparación constante entre ellas. La segmentación, separación, ordenación, búsqueda y recuperación de datos se realiza mediante el programa informático Atlas-ti.

\section{Resultados del estudio}

En este epígrafe presentamos los principales hallazgos obtenidos de uno de los estudios de casos realizados. Nos aproximaremos al modo particular que sigue un profesor iniciado o intermedio, de 6-15 años de experiencia docente, valorado como "buen docente", perteneciente al área de las Ciencias Sociales y Jurídicas y que desarrolla su actividad de enseñanza en contextos presenciales y no presenciales, para elaborar un conocimiento docente que le permita intervenir en escenarios formativos, adaptar y transformar los saberes disciplinares disponibles en representaciones didácticas que hagan comprensible el contenido de enseñanza a los estudiantes. El sistema de registro utilizado para citar las unidades de significado que sustentan las interpretaciones realizadas por el investigador incluyen una referencia numérica mediante la cual identificamos al profesor (P1). En el caso de los fragmentos extraídos de la entrevista biográfica nos referimos a EB y para la entrevista de estimulación del recuerdo a EER.

Particularmente, estamos ante un docente que se ha especializado en un campo específico, participando en líneas de trabajo bien definidas a través de proyectos, estancias de investigación, publicaciones científicas y actividades de gestión. Se define 
claramente como un profesor con una intensa formación disciplinar desarrollada con el devenir del tiempo y consideración menor de la formación pedagógica, dado que cuando ha debutado eran casi inexistentes los dispositivos formales de formación pedagógica. En este sentido, encontramos un claro predominio de fuentes de aprendizaje no formal, entendido como un proceso mediante el cual se adquiere conocimiento en el lugar de trabajo y sin un apoyo intencional y sistemático.

Estamos ante un docente que reconoce una escasa influencia del aprendizaje entre iguales en su trayectoria profesional, pero que tiene un afán marcado por conectar la materia con la experiencia de vida y hacerla atractiva a sus estudiantes.

$\mathrm{Si}$, básicamente de lo que partí es intentar vincular las explicaciones con experiencias de la vida de los estudiantes, es un poco lo que intento hacer, que vean que tenemos una materia que no es ajena, sino que tiene puntos de conexión con lo que hacen habitualmente, con las noticias que leen ¿no?, sería un poco la idea (EBP1, p.9)

Mantiene, pues, una constante postura reflexiva e introspectiva sobre su tarea pedagógica. Su quehacer está caracterizado por una triple y genuina preocupación: relación dialéctica entre teoría y práctica, la planificación y coordinación docente Presenta, ciertamente, un patrón de actuación bastante definido.

...combinar contenidos teóricos con contenidos prácticos, yo creo que es importante, sistematizar eso que vemos en la práctica con contenidos teóricos, es decir darles una cierta estructura, es decir, esto es así y así y lo vemos en este ejemplo, pero que ellos tengan también este armazón teórico que es lo que les puede servir también después en una práctica profesional para intentar extraer digamos para su caso concreto otras soluciones ¿no? (EERP1, p.14-15)

La comprensión en el alumnado, la dimensión dialógica reflexiva (inédita hasta ahora en los estudios sobre el CDC en la educación superior) y la transformación del saber (adaptaciones del conocimiento científico que el profesor realiza para que se adecúe al alumnado y a sus conocimientos previos) aparecen aquí como indicadores del CDC en la práctica. En este caso, se evidencian a través del discurso del profesor que tiene el propósito de explicar, exponer, aclarar, concretar, asociar, destacar, preguntar y relacionar conceptos, hechos, procedimientos, problemas, etc. y el texto utilizado en el aula que sirve de soporte, guía o síntesis de la exposición de los contenidos. El uso de materiales para la enseñanza se visualiza como un elemento que cobra significado, mediando en los procesos de enseñanza y aprendizaje y adoptando la función específica de facilitar la organización y exposición de contenidos. Su elaboración representa 
una manifestación clara del CDC y actúa como indicador su construcción. Para el docente en cuestión los materiales auténticos (ejemplificaciones de sentencias, impresos de carácter institucional, artículos de prensa, etc.) tienen la función de facilitar la organización y exposición de contenidos. Actúan, de alguna manera, como un elemento vertebrador, buscando relacionar el contenido con cuestiones de actualidad y con la futura práctica profesional de los discentes.

...muy presente en toda su actividad profesional opten por lo que opten, es decir, incluso si optan por asesorar a una empresa, la empresa tiene constantemente trato con una administración, entonces un poco pues de acercar al funcionamiento diario de la sociedad la materia que explico quizás... (EERP1, p.14)

Las presentaciones digitales, particularmente, actúan como guías estructurantes del contenido. El aula virtual como repositorio de materiales.

Como repositorio de materiales. Colgar materiales y que los cojan y nada más, son muchos alumnos con lo cual dificulta un poco (EBP1, p. 12)

Tanto el discurso como el texto nos aproximan a lo que algunos entienden como "configuraciones didácticas". Es decir, a aquellas maneras particulares que el profesor despliega para favorecer los procesos de construcción y apropiación del conocimiento.

En el patrón docente emerge la necesidad de atender a las expectativas de comprensión en el alumnado. En su caso particular, está presente fundamentalmente en las acciones didácticas relacionadas con la organización de los contenidos de la materia. Se pone de manifiesto a través de estrategias tales como: recapitulación y anticipación de contenidos, integración disciplinar, conflicto cognitivo, acciones de salida y de cierre. La anticipación y recapitulación de contenidos de enseñanza, preparar para aprender, tienen como propósito despertar la curiosidad en el alumnado, establecer prerrequisitos de comprensión, generar dinamismo en las temáticas de estudio, mostrar las diferencias entre lo que saben y lo que deberían saber, etc.

Yo hago recapitulaciones, eso sí lo suelo hacer también, tiendo a intentar establecer un poco un camino, tiendo a establecer como un camino y lo hago a principio de curso, es decir, estamos explicando el derecho administrativo III, pues antes explicamos el I, el II y decidimos ordenar la materia así porque en el I explicamos esto por este motivo, en el II esto y el III va después por estos dos motivos ¿no? (EERP1, p.15)

Decir un poco dónde estamos, a dónde vamos, de dónde venimos (EERP1, p.16) 
La integración disciplinar busca la relación entre el contenido de la materia y otras materias afines, promoviendo las interrelaciones entre saberes disciplinares.

"Esto hace referencia al curso pasado, ya que vieron lo de impulso de oficio en el curso pasado, tiendo a procurar relacionar con cosas que vieron, normalmente no se acuerdan de nada pero sí que procuro pues en servicios públicos si vieron contratos la semana anterior entonces la gestión contractual de servicios públicos, o sea intento hacer referencias a cosas que deben saber para que relacionen contenidos de la materia, no sabes sólo procedimientos, sólo no sé qué, todo está relacionado y todo va a ser necesario, pues a la hora de hacer una demanda pues tienes que conectar todo lo que sabes y partes más procesales y partes más materiales de y entonces necesitas" (EERP1, p.18)

El conflicto cognitivo persigue el planteamiento de situaciones problemáticas a través de situaciones reales que requieren un análisis y juicio del alumnado, quizás sea el indicador que pone de manifiesto la verdadera naturaleza del CDC (dinámica, compleja, situada y contextual).

...la ley manifiesta opciones políticas, opciones ideológicas, construye la sociedad de una determinada manera en función de una serie de intereses y eso quiero que lo vean en clase, entonces a veces también un poco les planteo: ¿cómo haríais esto?, ¿cómo regularíais esto?, un poco se ve también en esos casos, en lo de la niña del velo (EERP1, p.23)

Las acciones de salida se definen como estrategias que tienen lugar al comienzo de la clase y que sintetizan las ideas clave trabajadas en una sesión de clase o abordan cuestiones organizativas, un indicador habitual en el patrón docente de nuestro estudio de caso. Las acciones de cierre, se conceptualizan como estrategias que permiten cerrar una secuencia didáctica. El humor y las historias anecdóticas se utilizan también, ocasionalmente, como estrategias para motivar y reforzar las explicaciones en el aula.

Vamos a ver, lo que sí que intento es que haya un acercamiento más informal a los estudiantes, en general yo creo que en mi facultad el profesorado es bastante rígido y clásico, distante y eso tampoco me gusta, tampoco me gusta el compadreo con los estudiantes, es decir, no busco al salir de clase ser la colega que va a tomar un café con un estudiante y no se me ocurre y nunca lo hago y sí veo que hay algún otro compañero que está así siempre rodeado de alumnos, pero también no quiero establecer un trato distante, entonces a veces 
simplemente por el tipo de lenguaje que utilizas, la forma de tratarlos, es decir, pues hay compañeros que tratan de usted a los alumnos, los más frecuentes, entonces yo creo que eso da una distancia que no es necesaria. ¿Por qué voy a tratar de usted a los estudiantes? Entonces ese tipo de recursos sí que los utilizo a veces para hacer más cercana la... (EERP1, p.31)

Todas estas estrategias parecen evidenciar que estamos ante un docente con un fuerte compromiso con el aprendizaje del alumnado y bajo un enfoque de corte constructivista.

Las actuaciones del docente, objeto de estudio, revelan un profundo conocimiento de la materia. La formación teórica disciplinar, imprescindible para la enseñanza universitaria, constituye aquí un eje determinante en la construcción del CDC. También manifiesta una habilidad para buscar aquellas estrategias más adecuadas para la enseñanza, por lo que podemos decir que, al tiempo, desarrolla un conocimiento específico que se refiere a la forma de enseñar la materia. En este caso, en el patrón docente se evidencian estrategias basadas en procesos dialógicos como pueden ser las preguntas reflexivas para generar hipótesis de comprensión o las propuestas de re-significación del contenido (interrogación didáctica, diálogo reflexivo, interpretación didáctica, etc.). La interrogación didáctica se puede entender como una especie de conversaciones socráticas que se manifiestan mediante el encadenamiento de interrogantes, guiando el pensamiento del estudiantado hacia el descubrimiento de las ideas y/o procedimientos que el docente desea mostrar.

La interpretación didáctica se define como una estrategia dirigida a la interpretación de las hipótesis de comprensión y de las preconcepciones que el alumnado tiene de la materia, donde el docente mediante preguntas reflexivas se pone en el lugar del estudiante identificando posibles dificultades o disposiciones de aprendizaje y buscando mejores modos de exponer el contenido. Existe, pues, una apuesta clara por una cultura de la práctica de enseñanza que privilegia el pensar en el aula. La interrogación didáctica y los ejemplos constituyen las estrategias más habituales en su patrón de actuación. En el caso del uso de los ejemplos, pensados para ilustrar o mostrar situaciones, fenómenos, etc., se manifiesta una relación inexcusable entre docencia e investigación.

...cada vez que tienes que explicar la potestad sancionadora y estás viendo en el caso del velo pues que están intentando sancionar sin un procedimiento sancionador, digamos, sin las garantías tal, pues te permite explicar la teoría y decir y esto fijaros aquí como está pasando, es decir, permite que esos contenidos teóricos se vean de forma más gráfica (EERP1, p.5) 
...después yo creo que es importante la labor investigadora para la docencia cosa que no es para la universidad. Esta idea de segmentar al profesorado y que exista profesorado investigador y docente, la idea es que exista profesores que no investiguen y solo sean docentes, es decir que estampen un manual y se lo salten a los alumnos y yo creo que eso resta mucho, yo una parte importante de las cosas que uso en clase son cosas que las uso por mi experiencia investigadora... (EBP1, p. 15)

Esta relación dialéctica posibilita la proximidad entre temáticas y herramientas utilizadas en una y otra faceta del trabajo docente universitario, la actualización en el campo de conocimiento y enriquece las manifestaciones del CDC en la práctica docente, si bien es cierto esta relación está condicionada por las situaciones institucionales particulares.

En definitiva, las acciones de aula se visualizan como trazos de saberes disciplinares y pedagógicos que se interrelacionan para dar forma y sentido didáctico a la materia de estudio. Parece que estamos ante un proceso que responde a un tipo de razonamiento pedagógico, en ocasiones de carácter intuitivo, que permite al docente reorganizar y transformar los saberes profesionales de los que dispone para la enseñanza de la asignatura.

Se identifica una relación estrecha, dinámica y permanente entre las manifestaciones del CDC en la práctica, la trayectoria biográfica y la formación pedagógica recibida. Algunas de las decisiones curriculares que toma tienen origen en las tres dimensiones citadas. Aquí se comprueba la importancia y validez de incorporar en el estudio del CDC tanto la dimensión biográfica como la del contexto institucional.

\section{Conclusiones finales}

Los datos arrojados por el estudio de caso presentado caracteriza una docencia basada en el aprendizaje del alumnado y en la consecución de competencias profesionales específicas y bajo hábitos pedagógicos que entran en tensión con aquellos generalizados hasta ahora en nuestras universidades: el empleo abusivo de la lección magistral, la escasa atención personalizada al alumnado, el trabajo individualizado y el énfasis en una enseñanza fragmentada, con una fuerte tendencia a la especialización y olvidada de los saberes humanísticos y de otras capacidades como trabajar en equipo. En este sentido, abunda en la idea de que ubicar al alumnado en el centro del proceso educativo implica trasladar la responsabilidad de "enseñar" del docente al "aprender" del alumno, aspecto que supone una transformación en la metodología, programación y evaluación de las asignaturas, pero sobre todo un cambio en las actitudes y en las concepciones docentes del profesorado. 
El profesorado universitario, según los hallazgos que se desprenden de nuestro estudio de caso, parece que deja de ser mero transmisor de conocimientos para convertirse en guía, organizador, supervisor o gestor. Es decir, el docente dedica una parte importante de su actividad a guiar y orientar al estudiante en su itinerario formativo, principalmente académico, pero también profesional y personal. Y la formación del estudiante se abre a una multitud de espacios y recursos curriculares sincrónicos y asincrónicos hasta el momento apenas contemplados en las prácticas de la enseñanza universitaria. Todo ello, exige al profesorado adaptar y transformar su conocimiento a una gran diversidad de metodologías y alternativas que, de manera combinada, conduzcan al alumnado hacia los nuevos objetivos de aprendizaje. Estudiar el CDC en el profesorado universitario, como es nuestro caso, arroja luz sobre cómo realizar estas transformaciones puesto que esos procesos de "transposición didáctica" (Chevallard, 1991) son precisamente su objeto.

Sin lugar a dudas, parece que poseer tanto un cierto dominio de teorías y estrategias pedagógicas y metodológicas que permitan desarrollar la enseñanza de manera efectiva como examinar de manera crítica la práctica para redefinir la acción en el aula e ir creciendo a nivel profesional constituyen aspectos relevantes. E, incluso, nos atrevemos a decir que para afrontar la práctica de la enseñanza universitaria se hace necesario el dominio de unas competencias clave (habilidades de planificación, metodología, motivación, comunicación, gestión del aula, evaluación, etc.) como plantea Marcelo (1999). La profesión docente, de cualquier nivel educativo, por lo tanto, es una profesión específica que requiere de una serie de habilidades, conocimientos, destrezas y actitudes particulares, propias y diferentes a las de otras profesiones.

EI CDC, como hemos podido visualizar en el estudio de caso, es un constructo muy robusto porque subsume y relaciona, de manera viva, dinámica y abierta, las dimensiones disciplinares y pedagógicas de la docencia universitaria e implica la capacidad del docente de encontrar nuevas representaciones del contenido que tengan virtualidad didáctica, fruto de un largo proceso y que algunos autores sitúan como propio de los docentes expertos como es el caso de nuestro sujeto de investigación y como hemos podido constatar en otros trabajos (Jarauta y Medina Moya, 2012). Es importante destacar que ese conocimiento no consiste únicamente en disponer de un elevado número de ejemplos, analogías o representaciones, sino que está caracterizado por un razonamiento pedagógico que facilita la generación de aquellas transformaciones.

A nuestro modo de ver, este estudio de caso puede ofrecer pistas y soluciones para responder a las demandas que genera el EEES. Sus hallazgos pueden ayudar a paliar la incapacidad de los programas de formación del profesorado universitario para dar respuestas formativas pertinentes a las situaciones ambiguas, inestables y complejas que caracterizan la enseñanza superior. En definitiva, las representaciones que los 
profesores universitarios bien valorados hacen de su enseñanza pueden extrapolarse a los programas de formación del profesorado.

Estudiar de manera integral las interconexiones entre los saberes pedagógicos y disciplinares puede contribuir al desarrollo de una enseñanza de calidad en algunos de los campos disciplinares de la educación superior. Bajo nuestro punto de vista los resultados de este trabajo de investigación pueden ayudar a consolidar en nuestro país los esfuerzos pioneros y a difundir casos de "buenas prácticas" entre la comunidad universitaria. Pero más allá de una diseminación de la mera descripción de buenas prácticas pensamos que permite poner a disposición de la comunidad universitaria algunas de las claves teóricas y operativas que permitan la excelencia en la enseñanza universitaria que, según nuestro estudio de caso, parece depender más bien de un proceso de construcción personal, motivado en un contexto profesional, cultural e histórico concreto.

\section{Referencias Bibliográficas}

Acevedo, J.A. (2009). Conocimiento Didáctico del Contenido para la enseñanza de la Naturaleza de la Ciencia (I): el marco teórico. Revista Eureka sobre Enseñanza y Divulgación de las Ciencias, 6(1), 21-46.

Bain, K. (2006). Lo que hacen los mejores profesores universitarios. Valencia: Publicacions Universitat de València.

Cruz Tomé, M.A. (2003). Necesidad y objetivos de la formación pedagógica del profesor universitario. Revista de Educación, 331, 35-66.

Chevallard, Y. (1991). La transposición didáctica. Del Saber Sabio al Saber Enseñado. Buenos Aires: AIQUE.

Doyle, W. (1990). Themes in teacher education research. En R. Houston (Ed.). Handbook of research of teacher education (pp. 3-24). Nueva York: MacMillan.

Evertson, C. y Green, J. (1989). La observación como indagación y método. En M. Wittrock (Comp.). La investigación de la enseñanza II. Métodos cualitativos y de observación (pp. 249-303). Barcelona: Paidós.

Feixas, M. (2002). El desenvolupament professional del professor universitari com a docent. Tesis doctoral. Departamento de Pedagogía Aplicada, Universidad Autónoma de Barcelona.

Glaser, B. y Strauss, A.L. (1967). The Discovery of Groundel Theory: strategies for Qualitative Research. Chicago: Aldine.

Goetz, J.P. y Lecompte, M.D. (1988). Etnografía y diseño cualitativo en investigación educativa. Madrid: Morata.

Hativa, N. y Goodyear, P. (Eds.) (2003). Teacher Thinking, Beliefs and Knowledge in Higher Education. Dordrecht: Kluwer. 
Jarauta, B. y Medina Moya, J.L. (2012). Fuentes y procesos de aprendizaje docente en el contexto universitario. Revista Española de Pedagogía, 252, 337-353.

Jarauta, B., Medina Moya, J. L. y Mentado, T. (2016). La transformación del saber en la enseñanza universitaria. Una aproximación desde el estudio del CDC. Revista de Investigación Educativa, 34(2), 471-485 [Disponible en http://dx.doi.org/10.6018/rie.34.2.221711].

Kember, D. (1997). A reconceptualisation of the research into university academic's conceptions of teaching. Learning and instruction, 7(3), 255-275.

Knight, P. (2005). El profesorado de educación superior. Formación para la excelencia. Madrid: Narcea.

Leite Ramalho, B. y Beltrán Llavador, J. (2012). Universidad y sociedad: la pertinencia de educación superior para una ciudadanía plena. Revista Lusófona de Educação, 21, 33-52 [Disponible en http:// revistas.ulusofona.pt/index.php/rleducacao/issue/view/234].

Lueddeke, G. (2003). Professionalising Teaching Practice in Higher Education: a study of disciplinary variation and 'teaching-scholarship'. Studies in Higher Education, 28 (2), 213-228.

Marcelo, C. (1993). Como conocen los profesores la materia que enseñan. Algunas contribuciones de la investigación sobre conocimiento didáctico del contenido. En L. Montero y J.M. Vez (Eds.). Las didácticas específicas en la formación del profesorado (I) (pp. 151-186). Santiago de Compostela: Tórculo.

Patton, M. (1987). How to use qualitative methods in evaluation. Beverly Hills: Sage.

Shulman, L.S. (2005). Conocimiento y enseñanza: fundamentos de la nueva reforma. Profesorado. Revista de currículum y formación del profesorado, 9(2), 1-31.

Stake, R.E. (1998). Investigación con estudio de casos. Madrid: Morata.

\section{$M^{a}$ Dolores Fernández Tilve}

Profesora del Departamento de Pedagogía y Didáctica y Coordinadora del

Grupo de Investigación ATALAIA

de la Universidad de Santiago de Compostela (USC).

Su actividad docente y de investigación está estrechamente ligada al campo de la formación y el desarrollo profesional del profesorado.

Email: mdolores.fernandez.tilve@usc.es ORCID : http://orcid.org/0000-0002-5047-8913

Correspondência:

$M^{a}$ Dolores Fernández Tilve

Universidad de Santiago de Compostela (USC), Facultad de Ciencias de la Educación, Campus Vida, S/N 15782 Santiago de Compostela, A Coruña España

Data de submissão: Dezembro 2018

Data de avaliação: Março 2019

Data de publicação: Setembro 2019 